\title{
Funcionamento da oxigenoterapia hiperbárica e seu uso no tratamento do pé diabético: quais os cuidados de enfermagem?
}

Functioning of hyperbaric oxygen therapy and its use in the treatment of diabetic foot: what nursing care?

Funcionamiento de la oxigenoterapia hiperbárica y su uso en el tratamiento del pie diabético: ¿qué cuidados de enfermería?

Sabrina Iracema da Silva Couto ORCID: https://orcid.org/0000-0002-3864-4843 Universidade Maurício de Nassau, Brasil E-mail: sabrinacouto89@gmail.com

Dayana Ruthyally da Rocha Silva ORCID: https://orcid.org/0000-0003-1982-0482 Universidade Maurício de Nassau, Brasil E-mail: ruthyallyd@gmail.com

Ester Teixeira Lopes ORCID: https://orcid.org/0000-0002-9894-5760 Universidade Maurício de Nassau, Brasil E-mail: ester17teixeira@gmail.com

Bianca Kaline Ferreira Torres ORCID: https://orcid.org/0000-0003-2792-1398 Universidade Maurício de Nassau, Brasil

E-mail: biancakaline10@gmail.com

Maria Gabriela de Oliveira Frazão ORCID: https://orcid.org/0000-0002-5223-3493 Universidade Maurício de Nassau, Brasil E-mail: gabriela.oliveirafz@gmail.com

Raquel Maria da Silva

ORCID: https://orcid.org/0000-0002-0214-6017 Universidade Maurício de Nassau, Brasil

E-mail: raquelsilvaraquel2016@hotmail.com Danielly Danubia da Silva ORCID: https://orcid.org/0000-0002-5396-8002 Universidade Maurício de Nassau, Brasil

E-mail: daniellydanubia2016@outlook.com

Maria da Conceição Ferreira de Lima ORCID: https://orcid.org/0000-0002-7259-320X Universidade Maurício de Nassau, Brasil E-mail: ceca1lima@hotmail.com

Joana Barbosa Ferreira da Silva ORCID: https://orcid.org/0000-0002-1586-0156 Universidade Maurício de Nassau, Brasil E-mail: joanabfsm@gmail.com Suêrda Izabela da Silva ORCID: https://orcid.org/0000-0002-8290-4817 Universidade Maurício de Nassau, Brasil E-mail: suerdaizabella@outlook.com Alandeivid Ramos Ferreira ORCID: https://orcid.org/0000-0003-1738-6520 Universidade Maurício de Nassau, Brasil E-mail: alandeividferreira2@hotmail.com

Alexsandro da Silva Lima

ORCID: https://orcid.org/0000-0001-5378-277X Universidade Maurício de Nassau, Brasil E-mail: allexsillvallima@hotmail.com

Débora Maria das Dores Alves ORCID: https://orcid.org/0000-0003-1432-5917 Universidade Maurício de Nassau, Brasil E-mail: deboraalves1@outlook.com 


\title{
Resumo
}

Objetivo: compreender o funcionamento da Oxigenoterapia Hiperbárica, seu uso no tratamento do pé diabético e os cuidados de enfermagem necessários. Metodologia: Trata-se de uma revisão integrativa da literatura, realizada no mês de agosto de 2021, nas bases de dados Biblioteca Virtual em Saúde (BVS), Literatura Latino-Americana e do Caribe em Ciências da Saúde (LILACS), Scientific Electronic Library Online (SCIELO) e Google Scholar, foram utilizados artigos escritos na língua portuguesa, publicados no período entre 2017 e 2021. Resultados: Foram encontrados 120 artigos no total, após rigor metodológico por meio dos critérios de inclusão e exclusão, foram selecionados 14 artigos, sendo 1 artigo na BVS, 1 na Lilacs, 0 na Scielo e 12 no Google Scholar. A análise dos artigos selecionados foi feita de forma descritiva e predispôs a etapa de extração dos dados: base de dados, autores/ano de publicação, periódico/tipo de estudo, título e temática. Conclusão: Diante dos resultados encontrados na literatura pode-se observar que a Oxigenoterapia Hiperbárica é uma importante aliada no tratamento de feridas, se provando positiva para o tratamento da síndrome do pé diabético e para ser realizada com êxito, é fundamental que os profissionais de enfermagem realizem todos os cuidados necessários, no sentido de evitar complicações inerentes ao meio, durante e depois das sessões, contribuindo para que se obtenham os melhores resultados.

Palavras-chave: Oxigenação hiperbárica; Pé diabético; Cuidados de enfermagem.

\begin{abstract}
Objective: understand the functioning of Hyperbaric Oxygen Therapy, its use in the treatment of diabetic foot and the necessary nursing care. Methodology: This is an integrative literature review, carried out in August 2021, in the Virtual Health Library (VHL), Latin American and Caribbean Literature in Health Sciences (LILACS), Scientific Electronic Library databases Online (SCIELO) and Google Scholar, articles written in Portuguese, published in the period between 2017 and 2021, were used. Results: A total of 120 articles were found, after methodological rigor through the inclusion and exclusion criteria, 14 articles were selected, 1 article in the VHL, 1 in Lilacs, 0 in Scielo and 12 in Google Scholar. The analysis of selected articles was descriptive and predisposed to the data extraction stage: database, authors/year of publication, journal/type of study, title and theme. Conclusion: Given the results found in the literature, it can be observed that Hyperbaric Oxygen Therapy is an important ally in the treatment of wounds, proving to be positive for the treatment of diabetic foot syndrome and to be successfully performed, it is essential that nursing professionals perform all the necessary care, in order to avoid complications inherent to the environment, during and after the sessions, contributing to obtain the best results.
\end{abstract}

Keywords: Hyperbaric oxygenation; Diabetic foot; Nursing care.

\section{Resumen}

Objetivo: abordar el contexto del uso de la Oxigenoterapia Hiperbárica en el tratamiento del pie diabético y los cuidados de enfermería necesarios. Metodología: Se trata de una revisión integradora de la literatura, realizada en agosto de 2021, en la Biblioteca Virtual en Salud (BVS), Literatura Latinoamericana y del Caribe en Ciencias de la Salud (LILACS), Bases de datos de la Biblioteca Electrónica Científica en Línea (SCIELO) y Google Scholar, artículos redactados en portugués, publicados en el período entre 2017 y 2021. Resultados: Se encontraron un total de 120 artículos, luego de rigor metodológico a través de los criterios de inclusión y exclusión, se seleccionaron 14 artículos, 1 artículo en la BVS, 1 en Lilacs, 0 en Scielo y 12 en Google Scholar. El análisis de los artículos seleccionados fue descriptivo y predispuesto a la etapa de extracción de datos: base de datos, autores/año de publicación, revista/tipo de estudio, título y tema. Conclusión: A la vista de los resultados encontrados en la literatura, se puede observar que la Oxigenoterapia Hiperbárica es un aliado importante en el tratamiento de heridas, resultando ser positiva para el tratamiento del síndrome del pie diabético y para ser realizada con éxito, es fundamental que los profesionales de enfermería realizan todos los cuidados necesarios, con el fin de evitar complicaciones inherentes al entorno, durante y después de las sesiones, contribuyendo a obtener los mejores resultados.

Palabras clave: Oxigenación hiperbárica; Pie diabético; Atención de enfermería.

\section{Introdução}

A Oxigenação Hiperbárica é uma técnica terapêutica que surgiu em 1622 e se expandiu no século XIX, sendo muito usada para tratamento de enfermidades como a tuberculose, surdez, anemias, hemorragias e cólera, também sendo conhecida 
como Oxigenoterapia Hiperbárica (OHB). No Brasil, essa técnica começou a ser usada somente em 1930 e era restrita ao tratamento de mergulhadores com doenças descompressivas, porém só foi regulamentada no ano de 1995 pelo Conselho Federal de Medicina com a resolução 1.457/95 como modalidade terapêutica (da Cunha, de Araújo \& Adorno, 2019; Siona \& Verissimo, 2019; Ganilha, 2020).

A oxigenoterapia hiperbárica funciona baseada em duas leis da física, sendo a Lei de Boyle-Mariotte que enfatiza que o aumento da pressão faz com que as cavidades que estejam fechadas sofram reversão, e a Lei de Henry que ressalta que quanto maior for a pressão de um gás sobre um líquido, maior será a solubilidade do gás sobre esse líquido. Na prática, o indivíduo submetido a esta terapia faz a inalação de oxigênio com concentração de 100\% dentro de uma câmara hiperbárica, onde a pressão ultrapassa 1 atmosfera absoluta (ATA). Dessa maneira, oferta-se uma grande quantidade de oxigênio na corrente sanguínea possibilitando a sua chegada aos diversos tecidos do corpo humano, permitindo assim o efeito terapêutico de ação de neovascularização, antimicrobiana, proliferação de fibroblastos, atividades osteoclásticas e osteoblásticas, auxiliando no processo de cicatrização das lesões (Neves \& Carvalho, 2020; da Cunha, de Araújo \& Adorno, 2019).

Para realização dessa terapia atualmente existe duas possibilidades de equipamentos hiperbáricos, sendo denominados de câmaras hiperbáricas, as quais podem ser monoplace (monopacientes) ou multiplace (multipacientes), que são diferenciadas pela capacidade de comportar números diferentes de pacientes. As câmaras do tipo monopacientes são destinadas para um único paciente por vez, o qual irá respirar oxigênio puro comprimido. Nas câmaras multipacientes, vários pacientes serão expostos ao ar pressurizado por meio de uma máscara facial, capa ou um tubo endotraqueal. As vantagens do primeiro tipo de câmara é que ela oportuniza um tratamento individualizado com pressurização fielmente recomendada, enquanto o segundo tipo de câmara fornece uma mesma ATA para todos os pacientes, desse modo, recomenda-se que sejam tratados pacientes com o mesmo tipo de lesão por sessão nelas (Alves, Gurgel, da Silva \& de Araújo França, 2020).

A utilização de OHB para tratamento de lesões vem ganhando cada vez mais espaço na medicina, principalmente nas afecções dos membros inferiores, como o pé diabético. As lesões cutâneas do pé, como as úlceras, as infeções e a gangrena, são as maiores causas de hospitalização por complicações da Diabetes Mellitus (DM). Essas lesões são conhecidas como síndrome do pé diabético, que pode ser definida como uma condição neuropática facilitada por traumatismo prévio, devido à perda de sensibilidade, que geralmente ocorre com mais constância em pessoas que não têm os seus níveis de glicemia controlados, levando muitas vezes a amputação ou morte (Nunes, 2020; Vicente, 2017).

No Brasil, a síndrome do pé diabético acomete a população com frequência, causando grandes prejuízos na vida das pessoas doentes e de seus familiares, principalmente devido à repercussão psicológica, social e econômica, modificando as relações sociais e onerando o sistema público, caracterizando-se assim como um problema de saúde pública. É nesse contexto que os profissionais de enfermagem e sua equipe têm papel fundamental, pois precisam atuar no cuidado das lesões, articulando conhecimento e ação para que a assistência seja completa, e a oxigenoterapia hiperbárica é uma importante ferramenta no processo de cuidados com essas lesões, exigindo proeza de toda a equipe em sua administração, como recomenda as diretrizes vigentes (Scheck et al., 2019; de Lima et al., 2017). Dentro desse contexto, essa pesquisa se faz necessária, tendo por objetivo compreender o funcionamento da Oxigenoterapia Hiperbárica, seu uso no tratamento do pé diabético e os cuidados de enfermagem, bem como contribuir com a Literatura Brasileira.

\section{Metodologia}

Esta pesquisa consiste em uma revisão integrativa da literatura, que é um método usado para investigar estudos já existentes com intuito de se obter resultados sobre um determinado tema, de forma sintetizada e ordenada. Para elaboração desta revisão, foram utilizados os procedimentos metodológicos recomendados pela literatura vigente trabalhada, a saber: 1) Identificação do tema e da questão norteadora; 2) Estabelecimento de critérios de inclusão e exclusão; 3) Categorização dos 
artigos; 4) Avaliação dos resultados incluídos; 5) Interpretação dos resultados; 6) Síntese do conhecimento (Souza, Silva \& Carvalho, 2010; Crossetti, 2012; Botelho, de Almeida Cunha \& Macedo, 2011).

A criação e idealização deste estudo de revisão da literatura pautou-se na seguinte questão norteadora: Como a Oxigenoterapia Hiperbárica age no tratamento do pé diabético e quais os cuidados de Enfermagem nesse contexto?

Após rigor metodológico, as bases de dados utilizadas foram a Biblioteca Virtual em Saúde (BVS), Literatura LatinoAmericana e do Caribe em Ciências da Saúde (LILACS), Scientific Electronic Library Online (SCIELO) e Google Scholar. Para as bases de dados foram usados os seguintes Descritores em Ciências da Saúde (DeCS): "Oxigenação Hiperbárica”, "Pé Diabético" e "Cuidados de Enfermagem".

O levantamento foi realizado no mês de agosto de 2021, atendendo a alguns critérios de inclusão e de exclusão. Foram excluídos trabalhos incompletos, estudos em animais, ensaios teóricos, artigos que não cumpriam o período estipulado, relatos de experiência e trabalhos que não compreendiam a temática estudada. Foram incluídos na pesquisa apenas trabalhos completos, originais ou revisões da literatura, que atendam ao objetivo desta pesquisa, escritos na língua portuguesa, publicados no período entre 2017 e 2021. A forma de seleção descrita na metodologia pode ser observada na Figura 1.

Figura 1: Esquema da seleção dos artigos.

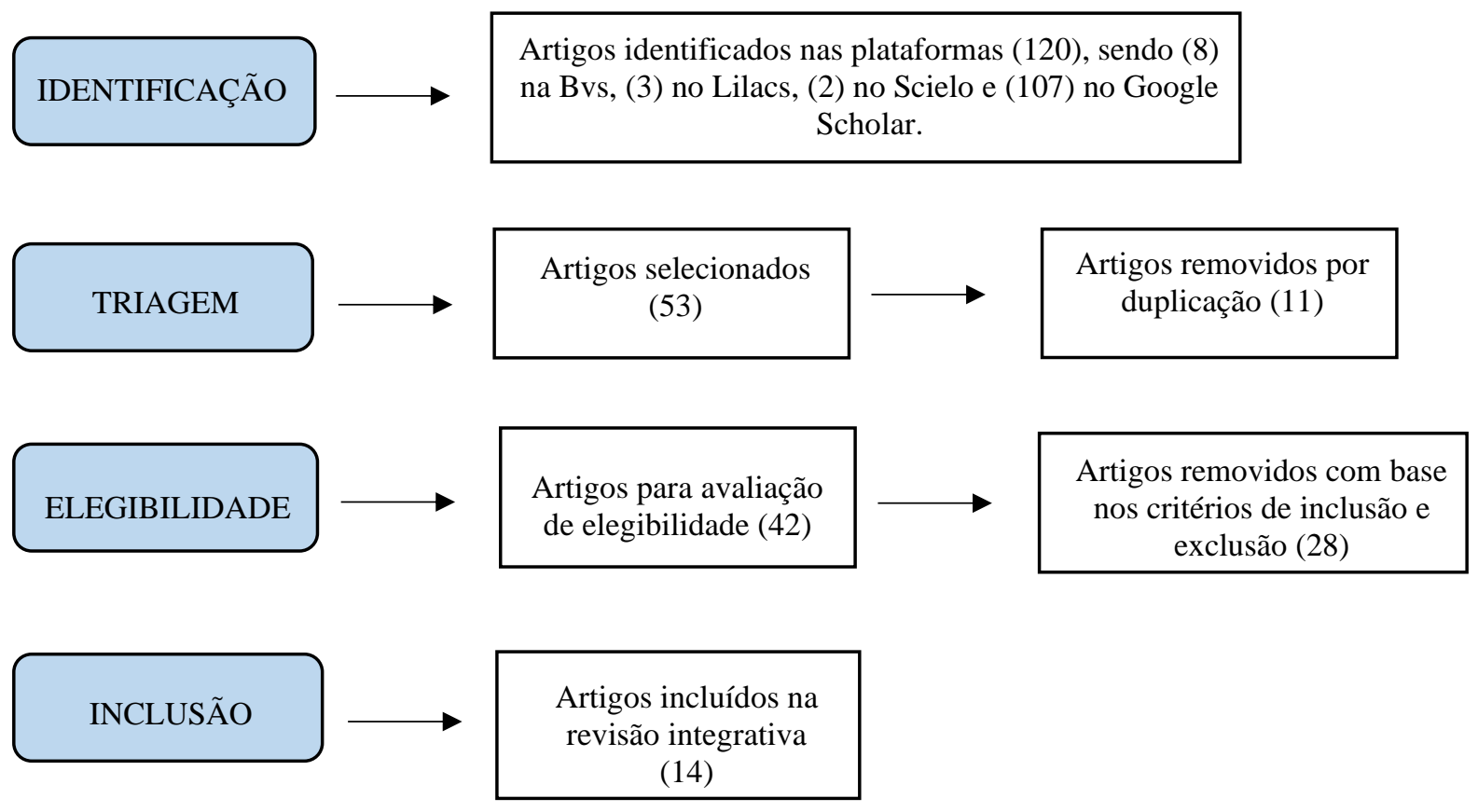

Fonte: Autores (2021).

\section{Resultados}

Foram encontrados 120 artigos no total, após rigor metodológico por meio dos critérios de inclusão e exclusão, foram selecionados 14 artigos, sendo 1 artigo na BVS, 1 na Lilacs, 0 na Scielo e 12 no Google Scholar. A análise dos artigos selecionados foi feita de forma descritiva e predispôs a etapa de extração dos dados: base de dados, autores/ano de publicação, periódico/tipo de estudo, título e temática (Quadro 1). 
Quadro 1 - Classificação dos artigos quanto base de dados, autor/ano de publicação, periódico/tipo de estudo, título e temática.

\begin{tabular}{|c|c|c|c|c|}
\hline $\begin{array}{c}\text { Base de } \\
\text { dados }\end{array}$ & $\begin{array}{l}\text { Autor / ano de } \\
\text { publicação }\end{array}$ & $\begin{array}{l}\text { Periódico / tipo de } \\
\text { estudo }\end{array}$ & Título & Temática \\
\hline $\begin{array}{l}\text { Google } \\
\text { Scholar }\end{array}$ & $\begin{array}{l}\text { Félix \& dos Santos } \\
\text { / } 2017\end{array}$ & $\begin{array}{l}\text { Revista Transformar / } \\
\text { Revisão da Literatura }\end{array}$ & $\begin{array}{lcr}\begin{array}{l}\text { Assistência de enfermagem } \\
\text { paciente }\end{array} \text { submetido } & \text { à } \\
\text { oxigenoterapia hiperbárica } & \end{array}$ & $\begin{array}{l}\text { Cuidados de Enfermagem na } \\
\text { Oxigenoterapia Hiperbárica }\end{array}$ \\
\hline $\begin{array}{l}\text { Google } \\
\text { Scholar }\end{array}$ & $\begin{array}{l}\text { Alves, Gurgel, da } \\
\text { Silva \& de Araújo } \\
\text { França / } 2020\end{array}$ & $\begin{array}{l}\text { Revista Enfermagem } \\
\text { Atual In Derme / } \\
\text { Revisão da Literatura }\end{array}$ & $\begin{array}{lcc}\text { Oxigenoterapia hiperbárica } & \text { no } \\
\text { processo de cicatrização } & \text { de } \\
\text { feridas: revisão de literatura } & \end{array}$ & $\begin{array}{l}\text { Funcionamento da Oxigenoterapia } \\
\text { Hiperbárica e sua aplicação no } \\
\text { tratamento de feridas }\end{array}$ \\
\hline LILACS & $\begin{array}{lr}\text { Liandro, } & \text { Santos, } \\
\text { de } & \text { Almeida } \\
\text { Carreiro, da Silva } \\
\text { Cunha \& de Paula } \\
\text { / } 2020\end{array}$ & $\begin{array}{l}\text { Revista Enfermagem } \\
\text { em Foco / Original }\end{array}$ & $\begin{array}{l}\text { Oxigenoterapia hiperbárica como } \\
\text { tratamento adjuvante para feridas: } \\
\text { estudo de prevalência }\end{array}$ & $\begin{array}{l}\text { Funcionamento da Oxigenoterapia } \\
\text { Hiperbárica e sua aplicação no } \\
\text { tratamento de feridas }\end{array}$ \\
\hline $\begin{array}{l}\text { Google } \\
\text { Scholar }\end{array}$ & $\begin{array}{l}\text { de Oliveira Lima } \\
\text { et al. / } 2020\end{array}$ & $\begin{array}{l}\text { Revista Eletrônica } \\
\text { Acervo Enfermagem / } \\
\text { Revisão da Literatura }\end{array}$ & $\begin{array}{llr}\text { Benefícios do } & \text { tratamento } & \text { com } \\
\text { oxigenoterapia } & \text { hiperbárica } & \text { em } \\
\text { úlcera venosa } & & \\
\end{array}$ & $\begin{array}{l}\text { Funcionamento da Oxigenoterapia } \\
\text { Hiperbárica e sua aplicação no } \\
\text { tratamento de feridas }\end{array}$ \\
\hline $\begin{array}{l}\text { Google } \\
\text { Scholar }\end{array}$ & $\begin{array}{l}\text { de Oliveira } \\
\text { Menezes, Cintra \& } \\
\text { Félix / } 2020\end{array}$ & $\begin{array}{ll}\text { Revista } & \text { Eletrônica } \\
\text { Acervo Saúde / } \\
\text { Revisão da Literatura }\end{array}$ & $\begin{array}{l}\text { Utilização da } \\
\text { hiperbárica no tratamento da } \\
\text { doença vascular periférica: uma } \\
\text { revisão sistemática }\end{array}$ & $\begin{array}{l}\text { Funcionamento da Oxigenoterapia } \\
\text { Hiperbárica e sua aplicação no } \\
\text { tratamento de feridas }\end{array}$ \\
\hline $\begin{array}{l}\text { Google } \\
\text { Scholar }\end{array}$ & $\begin{array}{l}\text { Scheck et al. / } \\
2019\end{array}$ & $\begin{array}{l}\text { Revista Enfermagem } \\
\text { Brasil / Original }\end{array}$ & $\begin{array}{l}\text { Práxis do enfermeiro e equipe de } \\
\text { enfermagem hiperbárica no } \\
\text { cuidado de pessoas com lesão de } \\
\text { pele }\end{array}$ & $\begin{array}{l}\text { Cuidados de Enfermagem na } \\
\text { Oxigenoterapia Hiperbárica }\end{array}$ \\
\hline $\begin{array}{l}\text { Google } \\
\text { Scholar }\end{array}$ & $\begin{array}{l}\text { da Cunha, de } \\
\text { Araújo \& Adorno / } \\
2019\end{array}$ & $\begin{array}{l}\text { Revista Feridas / } \\
\text { Revisão da Literatura }\end{array}$ & $\begin{array}{l}\text { Oxigenoterapia hiperbárica: } \mathrm{A} \\
\text { eficácia no tratamento de lesão em } \\
\text { pé diabético }\end{array}$ & $\begin{array}{l}\text { Oxigenoterapia Hiperbárica } \\
\text { tratamento do Pé Diabético }\end{array}$ \\
\hline $\begin{array}{l}\text { Google } \\
\text { Scholar }\end{array}$ & Nunes / 2020 & $\begin{array}{l}\text { Repositório UL / } \\
\text { Revisão da Literatura }\end{array}$ & $\begin{array}{l}\text { Oxigenoterapia hiperbárica: } \\
\text { princípios básicos e aplicação ao } \\
\text { pé diabético }\end{array}$ & $\begin{array}{l}\text { Oxigenoterapia Hiperbárica no } \\
\text { tratamento do Pé Diabético }\end{array}$ \\
\hline $\begin{array}{l}\text { Google } \\
\text { Scholar }\end{array}$ & Vicente / 2017 & $\begin{array}{l}\text { Repositório UBI / } \\
\text { Revisão da Literatura }\end{array}$ & $\begin{array}{lr}\text { A oxigenoterapia } & \text { hiperbárica } \\
\text { enquanto } & \text { terapêutica } \\
\text { complementar do pé diabético: útil } \\
\text { ou inútil? }\end{array}$ & $\begin{array}{l}\text { Oxigenoterapia } \text { Hiperbárica } \\
\text { tratamento do Pé Diabético }\end{array}$ \\
\hline $\begin{array}{l}\text { Google } \\
\text { Scholar }\end{array}$ & $\begin{array}{l}\text { Siona \& } \\
\text { Verissimo / } 2019\end{array}$ & $\begin{array}{l}\text { Repositório Faema / } \\
\text { Revisão da Literatura }\end{array}$ & $\begin{array}{l}\text { Uso de câmara hiperbárica para } \\
\text { tratamento de lesão por pressão: } \\
\text { atuação do enfermeiro }\end{array}$ & $\begin{array}{l}\text { Cuidados de } \\
\text { Oxfermagem na } \\
\text { Funcionoterapia Hiperbárica } \\
\text { Funcionento da Oxigenoterapia } \\
\text { Hiperbárica e sua aplicação no } \\
\text { tratamento de feridas }\end{array}$ \\
\hline $\begin{array}{l}\text { Google } \\
\text { Scholar }\end{array}$ & Leite Filha / 2019 & $\begin{array}{l}\text { Repositório Doctum / } \\
\text { Original }\end{array}$ & \begin{tabular}{llc} 
Eficiência da & \multicolumn{2}{c}{ oxigenoterapia } \\
hiperbárica & no tratamento de \\
úlcera venosa: estudo de caso
\end{tabular} & $\begin{array}{l}\text { Funcionamento da Oxigenoterapia } \\
\text { Hiperbárica e sua aplicação no } \\
\text { tratamento de feridas }\end{array}$ \\
\hline $\begin{array}{l}\text { Google } \\
\text { Scholar }\end{array}$ & $\begin{array}{l}\text { Neves \& Carvalho } \\
\text { / } 2020\end{array}$ & $\begin{array}{l}\text { Repositório } \\
\text { Unievangélica } \\
\text { Original }\end{array}$ & $\begin{array}{l}\text { Terapia hiperbárica e sua eficácia } \\
\text { nas lesões crônicas em uma clínica } \\
\text { particular do estado de goiás }\end{array}$ & $\begin{array}{l}\text { Funcionamento da Oxigenoterapia } \\
\text { Hiperbárica e sua aplicação no } \\
\text { tratamento de feridas }\end{array}$ \\
\hline $\begin{array}{l}\text { Google } \\
\text { Scholar }\end{array}$ & Lopes / 2019 & $\begin{array}{l}\text { Repositório Uniceub / } \\
\text { Revisão da Literatura }\end{array}$ & $\begin{array}{l}\text { Os efeitos da oxigenoterapia } \\
\text { hiperbárica adjuvante sobre as } \\
\text { úlceras do pé diabético: uma } \\
\text { revisão sistemática }\end{array}$ & $\begin{array}{l}\text { Oxigenoterapia Hiperbárica no } \\
\text { tratamento do Pé Diabético }\end{array}$ \\
\hline BVS & Silva / 2018 & $\begin{array}{l}\text { Revista Médica Minas } \\
\text { Gerais / Original }\end{array}$ & $\begin{array}{l}\text { Indicação de oxigenoterapia } \\
\text { hiperbárica como auxiliar na } \\
\text { cicatrização de úlceras de membro } \\
\text { inferiores }\end{array}$ & $\begin{array}{l}\text { Funcionamento da Oxigenoterapia } \\
\text { Hiperbárica e sua aplicação no } \\
\text { tratamento de feridas }\end{array}$ \\
\hline
\end{tabular}

Fonte: Autores (2021). 


\section{Discussão}

\subsection{Funcionamento da Oxigenoterapia Hiperbárica e sua aplicação no tratamento de feridas}

A oxigenoterapia hiperbárica, como já mencionado, foi normatizada no Brasil no ano de 1995 pelo Conselho de Medicina, através da resolução 1.457/95, passando então a ser reconhecida como modalidade terapêutica. A Sociedade Brasileira de Medicina Hiperbárica (SBMH), em 2003, estabeleceu com base nas diretrizes de segurança e qualidade, orientações para utilização e indicação desta terapia, visto que é necessário avaliação e liberação médica para sua realização (Liandro, Santos, de Almeida Carreiro, da Silva Cunha \& de Paula, 2020).

De acordo com Siona e Verissimo (2019) o desenvolvimento da linhagem da terapia hiperbárica aconteceu devido à exploração do ambiente subaquático, onde se procurava ultrapassar as barreiras por meio da utilização de equipamentos que tornassem aceitáveis o aumento do tempo e da profundez do mergulho. Desta forma, a Medicina Hiperbárica se dividiu em duas especialidades: uma condizia às atividades profissionais e saúde ocupacional dos mergulhadores, aeronautas e trabalhadores sob ar comprimido, e a outra se responsabilizava pela aplicação clínica da oxigenoterapia hiperbárica no espaço hospitalar, tendo por desafio estudar e sistematizar protocolos que confirmem a potencialidade clínica da terapia.

Deste modo, como antes já mencionado, dois tipos de câmaras hiperbáricas foram desenvolvidas para uso hospitalar: a monoplace, que adapta apenas uma pessoa que receberá inteiramente oxigênio puro pressurizado, e a câmara multiplace, que adapta mais pessoas em ambiente pressurizado, os quais receberão oxigênio puro ofertado por meio de máscaras faciais, capuz ou tubo endotraqueal. Valendo destacar que decorrente do modelo da câmara multiplace, é aceitável e recomendável a presença de profissionais de saúde para auxiliarem os pacientes que requeiram cuidados intensivos durante as sessões (Vicente, 2017; Siona \& Verissimo, 2019).

É importante reforçar que a OHB é uma técnica terapêutica onde o paciente recebe oxigênio puro a 100\%, em ambiente pressurizado, em um nível maior que a pressão atmosférica. A palavra hiperbárica deriva do grego hiper (excesso ou acima) e baros (pressão ou densidade). Nesse mecanismo, o oxigênio é responsável por um processo regenerativo mais acelerado que o convencional, alcançando resultados positivos no tratamento de diversas doenças, como embolias, infecções, gangrena, Síndrome de Fournier, infecções necrotizantes de partes moles, lesões por radiação, isquemias, queimaduras, vasculites, osteomielite e enxertos comprometidos (Félix \& dos Santos, 2017; Neves \& Carvalho, 2020; Silva, 2018).

Em relação ao mecanismo de funcionamento da OHB, os autores Alves et al. (2020) e de Oliveira Menezes, Cintra e Félix (2020), discorreram que a neovascularização humana é provocada a partir do aumento de células como VEGF (fator de crescimento endotelial vascular) e TGF- $\beta$ (fator de transformação do crescimento), células essas que são relacionadas ao fator de crescimento vascular, o que provoca a formação de novos capilares. Complementam ainda que durante as sessões de OHB, após receberem altas taxas de oxigênio, os tecidos sofrem um processo de hipóxia pós OHB, o que remete a estimulação da neovascularização.

Sabe-se que a pele é o maior órgão do corpo humano, sendo formada por uma dupla membrana que reveste todo o corpo, com a função principal de termorregulação, correspondendo a aproximadamente $16 \%$ do peso corporal. Por motivos de rompimento desse tecido, ou seja, perda de sua integridade, origina-se o que se chama de feridas ou lesões. Essas feridas podem ser classificadas por fatores que envolvem a causa, a origem microbiana, o tipo de cicatrização, o grau de abertura e o tempo de duração. Além do mais, as feridas nem sempre são facilmente tratadas, a depender do processo, as mesmas podem apresentar graves complicações, como por exemplo, as infecções que podem demandar correções cirúrgicas como a amputação de um membro. Nesse contexto, a OHB configura-se como um tratamento capaz de ofertar oxigênio com o objetivo de melhorar a hipoperfusão e a inflamação dos tecidos, comportando-se como uma droga que provoca a elevação do metabolismo (Leite Filha, 2019; Alves et al., 2020). 
Os autores Liandro et al. (2020) e de Oliveira Lima et al. (2020) complementaram com detalhes os efeitos físicos e metabólicos dessa oxigenoterapia no tratamento das feridas, por exemplo, o efeito antimicrobiano que é alcançado à medida que os níveis teciduais do oxigênio são elevados, diminuindo a atividade das endotoxinas. Ou o efeito de vasoconstricção proporcionada por essa pressão de oxigênio aumentada, que responde na redução do edema, a angiogênese devido ao efeito compensatório da hipóxia, aumento da matriz óssea que devido a esse aumento de oxigênio dissolvido nos líquidos teciduais, aumenta a chegada de oxigênio nos ossos que permite as atividades osteoclásticas e osteoblásticas. Logo, a oxigenoterapia vem ganhando espaço como terapêutica no tratamento de feridas, uma vez que, por estar centrada na cicatrização das lesões, não se restringe ao uso das coberturas e medicamentos. Valendo destacar que devido a necessidade de câmara hiperbárica para sua realização, seu custo é elevado, em razão de não ser uma tecnologia barata, custando em média 300 reais a sessão e todo o tratamento pode custar entre 1 mil a 18 mil reais, e não é facilmente ofertado pela rede pública de saúde, o que a torna não acessível para todos.

\subsection{Oxigenoterapia Hiperbárica no tratamento do Pé Diabético}

A diabetes mellitus (DM) é uma patologia crônica caracterizada pela elevação dos níveis de glicose no sangue (hiperglicemia), podendo ocorrer devido a defeitos na secreção ou funcionamento da insulina, que é um hormônio produzido pelas células beta do pâncreas. A DM apresenta uma grande prevalência na população mundial, manifestando alterações neurológicas, microvasculares e macrovasculares, desencadeando inúmeras complicações locais e sistémicas, como por exemplo, retinopatia, nefropatia, neuropatia e vasculopatia, com grande impacto na morbimortalidade dos doentes (Vicente, 2017).

Os doentes com DM são mais propícios a desenvolverem úlceras crônicas nos pés, devido à perda de sensibilidade característica da neuropatia associada a esta patologia. Ocorrendo geralmente em pacientes que não têm seus níveis de glicose controlados, que frequentemente apresentam também neuroartropatia de Charcot e osteomielite, constituindo o que se conhece por síndrome do pé diabético. Como consequência desta neuropatia, estas úlceras geralmente apresentam infeções, sendo que podem muitas vezes se manifestarem de forma assintomática, contribuindo assim na possibilidade de mais tempo sem nenhum tipo de tratamento, evoluindo rápido para a cronicidade (Nunes, 2020; Vicente, 2017).

Aprofundando o entendimento acerca do desenvolvimento da neuropatia na DM, o autor Lopes (2019) apresentou duas teorias, uma vascular e uma bioquímica. Na teoria vascular, uma microangiopatia isquêmica da vasa nervorum seria responsável pela lesão do nervo e suas respectivas manifestações clínicas. Já na teoria bioquímica, a elevação de elementos tóxicos, como sorbitol e frutose, associada à depleção do mioinositol, seria a responsável por lesar as células de Schwann e causar o quadro neuropático.

É importante frisar que para se classificar o estadiamento das ulcerações da síndrome do pé diabético, é recomendável a classificação de Wagner, que estratifica-as em 6 graus, sendo: Grau 0 - pé em risco, mas sem úlcera presente; Grau 1 - úlcera superficial, sem sinais clínicos de infeção; Grau 2 - úlcera mais profunda, que pode chegar a tendões, a cápsula articular ou ao osso; Grau 3 - envolvimento de tecidos, onde pode existir abcessos, osteomielite e tendinite; Grau 4 - gangrena localizada em apenas uma porção do pé; E por último, o Grau 5 - gangrena que envolve grande parte do pé, o suficiente para que apenas a amputação total seja a única opção (Nunes, 2020).

Deste modo, a oxigenoterapia hiperbárica tem sido proposta para diminuir internamentos e amputações associadas a esta doença, sendo a melhora clínica observada em alguns doentes através de alguns mecanismos fisiológicos, como a hiperoxigenação dos tecidos, ativação de fibroblastos, vasoconstrição, promoção de neovascularização em tecidos hipóxicos, efeitos antimicrobianos, ativação de fagócitos e neutrófilos (da Cunha et al., 2019; Nunes, 2020).

A principal explicação para uso da OHB nos diferentes tipos de úlceras do pé diabético é que, apesar das várias etiologias das feridas crônicas, o denominador comum parece ser a hipóxia. Pois em um balanço complexo entre a hipóxia da lesão e a 
oxigenação da região à sua volta, o sucesso da cicatrização depende de uma adequada oxigenação do tecido da periferia da lesão. No processo de cicatrização, a chegada insuficiente de oxigênio ao tecido pode fazer com que este processo não aconteça. Logo, a presença ainda que intermitente de oxigênio nestes tecidos hipóxicos pode permitir a retomada do processo normal de cicatrização. Desta forma, as sessões de OHB têm demostrado causar hiperoxigenação, vasoconstrição, ativação de fibroblastos, efeitos antimicrobianos, potenciação de antibióticos, redução da quimiotaxia de leucócitos, regulação de fatores de crescimento e de citocinas inflamatórias (Nunes, 2020; Lopes, 2019).

Em 2018, o Ministério da Saúde através da Portaria No 55, de 24 de outubro de 2018, tornou pública a decisão de incorporar a oxigenoterapia hiperbárica para tratamento do pé diabético, no âmbito do Sistema Único de Saúde (SUS), sem a necessidade de criação de procedimento específico e sem custo adicional (Brasil, 2018).

\subsection{Cuidados de Enfermagem na Oxigenoterapia Hiperbárica}

A enfermagem é uma profissão responsável por exercer diversas demandas dentro da saúde, envolvendo assistência, administração, ensino, pesquisa e participação política. Deste modo, é de competência do enfermeiro prestar os cuidados de maior complexidade técnica, que exijam conhecimentos de base científica e capacidade de tomar decisões, bem como administrar, coordenar sua equipe e dentre outros. Logo, o enfermeiro deve possuir preparo técnico e experiência para atender de forma necessária o paciente submetido à $\mathrm{OHB}$, acompanhando e observando a realização do procedimento, identificando possíveis efeitos colaterais e agindo de forma efetiva caso surjam (Félix \& dos Santos, 2017).

A Sociedade Brasileira de Medicina Hiperbárica recomenda que enfermeiros e técnicos de enfermagem operem e prestem cuidados aos pacientes submetidos a OHB, obedecendo a Lei no 7.498/86, que regulamenta o Exercício Profissional de Enfermagem. Os cuidados de enfermagem junto ao paciente são de extrema importância, pois é o profissional que dará continuidade na assistência à saúde, por meio de avaliação criteriosa do estado do paciente, acompanhando sua evolução e possíveis intercorrências que possam surgir causadas pela terapia. Ademais, o trabalho da equipe de enfermagem é essencial para prestar cuidado hiperbárico, visto que esses profissionais em uma unidade hiperbárica desempenharão cerca de $80 \%$ do trabalho (Siona \& Verissimo, 2019; Félix \& dos Santos, 2017).

Os autores Scheck et al. (2019) e Siona e Verissimo (2019) relataram que no contexto da organização do trabalho da enfermagem hiperbárica, o protagonismo e a atuação do enfermeiro só ocorreram a partir de 2008, visto que a OHB não fazia parte como especialidade na Resolução COFEN (Conselho Federal de Enfermagem) No 290/2004. No entanto, uma nova resolução foi revogada, a resolução COFEN No 577/2018 no que abrange o direito no currículo do profissional de enfermagem a especialidade hiperbárica. Mesmo diante dessa regulamentação, o entendimento da prática de enfermagem hiperbárica ainda é desconhecido pela maioria, sendo possível encontrar distanciamentos entre a produção do conhecimento e os processos necessários para uma prática e aprendizagem reflexiva pelo enfermeiro e equipe, gerando implicações em sua práxis, haja vista a lacuna científica de publicações brasileiras sobre a atuação da enfermagem em OHB.

Vale reforçar que a função do enfermeiro que atua na OHB é orientar o paciente sobre as medidas de segurança, acompanhamento e observação durante a terapia, monitorando os efeitos colaterais e estar preparado para fornecer suporte básico à vida, caso ocorram convulsões, acidentes, intoxicação pulmonar ou neurológica. Assim, a equipe de enfermagem assiste o paciente desde o encaminhamento, pré-terapia, trans-terapia e pós-terapia, cabendo a esses profissionais os seguintes cuidados: observar sinais e sintomas de barotrauma; orientar técnicas de equalização do ouvido como valsava, bocejar, deglutição e chiclete, encorajando o paciente para mesclar essas técnicas; notificar ao médico estado de ansiedade; parar a sessão em caso de queixa álgica; prevenir ou reduzir os efeitos da ansiedade causadas pelo confinamento; informar que pode sair quando necessário; remover o paciente da câmara nos casos de dor sem controle, ou intoxicação pelo oxigênio; notificar as dificuldades de 
equalização do ouvido para o operador da câmara; implementar intervalo de ar e monitorar a terapia hiperbárica (Félix \& dos Santos, 2017).

É imprescindível que o enfermeiro atue na OHB com foco na educação do paciente, acompanhamento e avaliação consecutiva do procedimento de cicatrização, bem como preparação do procedimento de tarefas, que necessita levar em conta as qualidades individuais, melhora do paciente durante o tratamento, avaliando sua potência e oferecendo assistência ao paciente durante toda a terapêutica (Siona \& Verissimo, 2019).

\section{Conclusão}

Em suma, as feridas ou lesões são patologias comuns no cotidiano de muitas pessoas, podendo apresentar complicações como infecções, amputação e morte. A Oxigenoterapia Hiperbárica é uma importante aliada no tratamento de feridas, uma vez que seus mecanismos de ação possibilitam o efeito terapêutico de neovascularização, ação antimicrobiana, angiogênese e regeneração, resultando no processo de cicatrização.

A síndrome do pé diabético também é uma patologia comum, atingindo uma população específica, os diabéticos. Como já mencionado, essas ulcerações crônicas nos pés ocorrem devido a perda da sensibilidade característica da neuropatia diabética, e como muitos diabéticos não têm seus níveis de glicose controlados, as complicações são recorrentes e agravantes. E a OHB se provou positiva e essencial para o tratamento da síndrome do pé diabético, visto que esta doença acarreta sérios problemas, sua utilização contribui na diminuição dos internamentos e amputações, dando melhores resultados e qualidade de vida.

Nesse contexto, para que a oxigenoterapia seja realizada com êxito, é fundamental que os profissionais de enfermagem realizem os cuidados necessários, como ter conhecimento das normas de segurança pessoal e de equipamentos, dos protocolos de tratamento dos pacientes, dos efeitos terapêuticos e adversos do oxigênio hiperbárico, a fim de guiar a avaliação da lesão e a implementação de um plano de cuidados efetivo em equipe, no sentido de evitar complicações inerentes ao meio, durante e depois das sessões, contribuindo para que se obtenham melhores resultados desta terapia.

Portanto, dentro do contexto da saúde, é primordial a constante evolução e atualização na assistência aos pacientes, permitindo assim fornecer cuidados de qualidade a todos. Inclusive deve-se lançar mão da OHB para potencializar esses cuidados de saúde, devendo os profissionais envolvidos, principalmente os de enfermagem, estarem devidamente capacitados para realizarem suas atribuições.

Desta forma, se faz necessário que estudos futuros sejam desenvolvidos nesta temática, visto que é um assunto importante para a saúde, que impacta diretamente no bem-estar de pacientes com feridas ou síndrome do pé diabético, que os mecanismos desta terapêutica precisam ser totalmente esclarecidos e conhecidos pelos profissionais, e especialmente porque o conhecimento e divulgação desse tema se encontra em escassez na literatura brasileira atualmente.

\section{Referências}

Alves, P., Gurgel, L. D. S. S., da Silva, V. D., \& de Araújo França, P. (2020). Oxigenoterapia hiperbárica no processo de cic atrização de feridas: revisão de literatura. Revista Enfermagem Atual In Derme, 93(31).

Botelho, L. L. R., de Almeida Cunha, C. C., \& Macedo, M. (2011). O método da revisão integrativa nos estudos organizacionais. Gestão e sociedade, 5(11), $121-136$.

Brasil (2018). Portaria No 55, de outubro de 2018. Ministério da Saúde / Secretaria de Ciência, Tecnologia e Insumos Estratégicos, ed. 206 , seção 1, 64. https://www.in.gov.br/materia/-/asset_publisher/Kujrw0TZC2Mb/content/id/47099524/do1-2018-10-25-portarian55-de-24-de-outubro-de-2018-47099388

Crossetti, M. D. G. O. (2012). Revisão integrativa de pesquisa na enfermagem o rigor científico que lhe é exigido. Revista gaúcha de enfermagem, 33(2), 8-9.

da Cunha, C. P. L., de Araújo, T. S., \& Adorno, W. C. L. D. (2019). Oxigenoterapia hiperbárica: A eficácia no tratamento de lesão em pé diabético. Revista Feridas, (39), 1414-1420.

de Lima, I. G., da Costa, J. F. L., de Oliveira, A. F., Junior, J. N. B., Peixoto, A. S., Pancieri, M. S., \& Pires, C. A. A. (2017). Educar para prevenir: a importância da informação no cuidado do pé diabético. Revista Conexão UEPG, 13(1), 186-195. 
Research, Society and Development, v. 10, n. 13, e241101320708, 2021

(CC BY 4.0) | ISSN 2525-3409 | DOI: http://dx.doi.org/10.33448/rsd-v10i13.20708

de Oliveira Lima, L., Marques, I. L. D. P. A., Nascimento, L. A. G., da Silva Nascimento, J., da Silva Schulz, R., David, R. A. R., \& Santa Rosa, D. D. O. (2020). Benefícios do tratamento com oxigenoterapia hiperbárica em úlcera venosa. Revista Eletrônica Acervo Enfermagem, 5, e4921-e4921.

de Oliveira Menezes, E., Cintra, B. B., \& Félix, V. H. C. (2020). Utilização da oxigenoterapia hiperbárica no tratamento da doença vascular periférica: uma revisão sistemática. Revista Eletrônica Acervo Saúde, 12(11), e5282-e5282.

Félix, R. A., \& dos Santos, R. A. (2017). Assistência de enfermagem ao paciente submetido à oxigenoterapia hiperbárica. Revista Transformar, $10,140-151$.

Ganilha, J. R. D. S. (2020). Frequência de convulsões hiperóxidas em mais de 150.000 sessões de oxigenoterapia hiperbárica: a experiência de um centro de medicina subaquática e hiperbárica (Doctoral dissertation). https://repositorio.ul.pt/bitstream/10451/42098/1/12273_Tese.pdf

Leite Filha, N. R. (2019). Eficiência da oxigenoterapia hiperbárica no tratamento de úlcera venosa: estudo de caso. Repositório Doctum, 1-22.

Liandro, C. L., Santos, M., de Almeida Carreiro, M., da Silva Cunha, K. C., \& de Paula, D. G. (2020). Oxigenoterapia hiperbárica como tratamento adjuvante para feridas: estudo de prevalência. Enfermagem em Foco, 11(2).

Lopes, V. B. (2019). Os efeitos da oxigenoterapia hiperbárica adjuvante sobre as úlceras do pé diabético: uma revisão sistemática. Repositório Uniceub, 1-36.

Neves, B. A., \& Carvalho, F. G. D. (2020). Terapia hiperbárica e sua eficácia nas lesões crônicas em uma clínica particular do estado de goiás. Repositório Unienvagélica, 1-53.

Nunes, R. J. A. (2020). Oxigenoterapia hiperbárica: princípios básicos e aplicação ao pé diabético. (Doctoral dissertation). Repositório UL, 04.

Scheck, V., Zmuda Padilha, D., Bonatto, C. R., de Oliveira Paz, P., Mallmann Duarte, Ê. R., \& Kaiser, D. E. (2019). Práxis do enfermeiro e equipe de enfermagem hiperbárica no cuidado de pessoas com lesão de pele. Enfermagem Brasil, 19(3).

Silva, A. C. D. (2018). Indicação de oxigenoterapia hiperbárica como auxiliar na cicatrização de úlceras de membro inferiores. Rev Med Minas Gerais, 28, e1967.

Siona, B. F., \& Verissimo, T. D. C. (2019). Uso de câmara hiperbárica para tratamento de lesão por pressão: atuação do enfermeiro. Repositório Faema, 1-37.

Souza, M. T. D., Silva, M. D. D., \& Carvalho, R. D. (2010). Revisão integrativa: o que é e como fazer. Einstein (São Paulo), 8(1), 102-106.

Vicente, R. D. S. (2017). A oxigenoterapia hiperbárica enquanto terapêutica complementar do pé diabético: útil ou inútil? (Doctoral dissertation). Repositório $U B I, 1-30$. 